\title{
Aux origines de l'esthétique barrésienne : le roman au miroir de la presse
}

\section{Séverine Depoulain}

\section{OpenEdition}

\section{$\checkmark$ Journals}

Édition électronique

URL : http://journals.openedition.org/edl/1001

DOI : 10.4000/edl. 1001

ISSN : 2296-5084

Éditeur

Université de Lausanne

\section{Édition imprimée}

Date de publication : 15 septembre 2017

Pagination : 49-64

ISBN : 978-2-940331-65-9

ISSN : 0014-2026

\section{Référence électronique}

Séverine Depoulain, «Aux origines de l'esthétique barrésienne : le roman au miroir de la presse »,

Études de lettres [En ligne], 2 | 2017, mis en ligne le 15 septembre 2019, consulté le 16 décembre 2020. URL : http://journals.openedition.org/edl/1001 ; DOI : https://doi.org/10.4000/edl.1001 


\section{AUX ORIGINES DE L'ESTHÉTIQUE BARRÉSIENNE: LE ROMAN AU MIROIR DE LA PRESSE}

Les liens étroits qui unissent les romans aux articles de presse à la Belle Époque invitent à reconsidérer la carrière littéraire de Maurice Barrès au regard de son statut de journaliste. Le romancier du Culte du moi et de l'Énergie nationale fait en effet partie d'une génération qui a su s'approprier les opportunités offertes par la presse pour penser la littérature. Études et chroniques littéraires forment ainsi un espace unique qui permet de comprendre la genèse de la sensibilité barrésienne.

"Journaliste déjà vieux, je me suis souvent éparpillé; c'était pour connaître mes limites et mes alentours " ${ }^{1}$. Ce constat, qu'établit Maurice Barrès dans le deuxième texte de Scènes et doctrines du nationalisme, résume les méandres d'une carrière journalistique aussi riche que complexe. Activité égotiste tout autant que tribune idéologique, l'écriture journalistique se conçoit, pour Barrès, comme une quête qui l'amène à explorer et à réconcilier les différentes facettes de son «moi». Lire les romans de Barrès en ayant à l'esprit ses articles de presse permet en effet de réaffirmer l'unité d'une œuvre littéraire, parfois perçue comme scindée en deux : à l'écrivain égotiste s'opposerait le romancier nationaliste. Si une évolution de la pensée et de l'esthétique barrésienne est à noter entre Le Culte du moi et Le Roman de l'énergie nationale, il est toutefois difficile de parler de véritable rupture. Plutôt qu'une transformation, la lecture des articles de presse de Barrès laisse entrevoir la maturation d'une pensée esthétique, stylistique et idéologique. Se présentant comme

I. M. Barrès, Scènes et doctrines du nationalisme, p. 8. 
un espace consacré à la création littéraire, le journal s'impose ainsi comme le lieu de la genèse de la sensibilité barrésienne.

C'est dès lors dans cette perspective qu'il est possible de considérer la poétique journalistique comme l'une des clefs d'entrée pour comprendre l'unité et la cohérence de l'œuvre barrésienne. Les premières chroniques de Barrès laissent en effet entrevoir le futur romancier à mesure que se dessinent un art littéraire et les thématiques récurrentes de son œuvre littéraire. Tout comme l'évolution de l'égotisme vers le nationalisme se lit dans les articles de presse que Barrès publie à l'époque.

\section{La presse, creuset de l'auvre romanesque barrésienne}

Héritier de Victor Hugo, de Balzac ou encore de George Sand, Maurice Barrès appartient à une longue lignée d'écrivains-journalistes qui envisagent le journalisme comme un formidable terrain d'expérimentation littéraire. L'écriture journalistique n'étant pas à l'époque nécessairement soumise à une déontologie de l'information, le recours à la fiction, à l'ironie ou encore au dialogue imaginaire se banalise sous la plume des journalistes de l'époque. Dès ses débuts, Maurice Barrès s'est approprié l'espace du journal pour former son art littéraire. Les travaux de Maurice Davanture ont, à ce sujet, mis en évidence les nombreuses similitudes qui peuvent être observées entre les premières chroniques de Barrès et ses romans de jeunesse.

Cette façon d'appréhender le journal comme un espace de création et d'inventivité littéraire s'exprime tout particulièrement dans les chroniques du Voltaire. Engagé par Alexandre Hepp en 1886 au terme de longues négociations, et après avoir collaboré à différentes petites revues littéraires, Maurice Barrès rejoint la rédaction de ce quotidien qui affiche sa volonté d'être «le Figaro des républicains». Le Voltaire souhaite en effet se démarquer d'autres périodiques, comme La Presse, par sa vivacité, son irrévérence et son esprit. Partisan du progrès et de la raison, il est le journal de l'homme libre. C'est dans cet esprit que Barrès y collabore pendant deux ans. Auteur de plus de quatre-vingts articles entre 1886 et $1888^{2}$, le jeune chroniqueur a la possibilité de publier tout ce qu'il écrit, sans peur d'être censuré. Le journal, dans lequel il publie des articles

2. Maurice Barrès collabore au Voltaire, du 10 mai 1886 au $1^{\text {er }}$ avril 1888. 
politiques, des chroniques littéraires ou encore des récits de voyage, devient le lieu d'expression du jeune écrivain en devenir. Utilisant l'actualité littéraire pour faire entendre différentes voix, Barrès joue avec les règles génériques de la chronique pour imposer son point de vue et sa manière au sein du champ littéraire.

Alors que Sous l'œil des Barbares est en cours d'écriture, le jeune chroniqueur utilise ainsi les opportunités offertes par ce quotidien pour exercer son style et sa pensée. Il s'agit non seulement de définir un regard face au monde mais également de traiter les thèmes qu'il développera tout au long de sa carrière. Le genre de la chronique, qui favorise l'emploi d'une écriture oblique, amène par exemple le jeune journaliste à perfectionner son art de l'ironie. Suivant avec précision le programme d'Émile de Girardin qui établit que la chronique est «le bulletin des livres nouveaux, des pièces en répétition, des modes nouvelles, des coutumes et usages nouveaux " ${ }^{3}$, Maurice Barrès adopte la désinvolture de l'ironiste pour décrire la société mondaine des années 1880 . La chronique consacrée à Dostoïevski lui permet de revenir avec humour sur l'effet de mode suscité par la littérature russe. Cette pratique régulière de l'ironie permet à Maurice Barrès de définir le point de vue qui sera le sien en tant que romancier. Comme l'explique Emmanuel Godo ${ }^{4}$, l'ironiste, en raison de son impossibilité à faire un avec le monde, développe un point de vue qui disloque et corrode le réel. Il montre ainsi que cette approche, qui rejoint la définition de l'ironie donnée par Jankélévitch, institue l'ironie comme méfiance et inattention au réel, mais également comme prélude à une confiance et à une attention sans borne. Utilisée de manière pragmatique afin de mettre en lumière les défaillances d'une certaine représentation du réel, l'ironie est un moyen qui permet d'accéder à une vérité plus complexe.

Ce double mouvement, observé par Emmanuel Godo, est déjà perceptible dans les chroniques du Voltaire. Le regard ironique du chroniqueur dévoile les ridicules et les failles des cercles littéraires dans lesquels il évolue pour mieux faire entendre une position plus personnelle. Par exemple, dans la chronique "Quinze cents métaphores» consacrée à Victor Hugo, les deux premières parties de la démonstration de Barrès sont placées sous le signe de l'ironie. Le journaliste décrit ainsi

3. É. de Girardin, La Presse, 21 septembre 1836.

4. E. Godo, La légende de Venise, p. 75. 
avec humour et détachement la démarche de Georges Duval qui s'est attaché à relever dans un dictionnaire les métaphores hugoliennes. Ce n'est qu'après ce préambule que Maurice Barrès s'autorise à proposer au lecteur sa propre lecture du texte hugolien. Reconnaissant le génie du poète de La Légende des siècles, et cela malgré le ridicule de certaines métaphores, le chroniqueur explique les raisons de son attachement et de ses réserves vis-à-vis de Victor Hugo. Une dernière pirouette sous forme de clin d'œil, où Maurice Barrès fait part de son souhait d'être "en règle vis-à-vis de [leur] grand lyrique national " ${ }^{5}$, clôt enfin la chronique. Certes, ces chroniques ne s'inscrivent pas encore dans la perspective d'une écriture égotiste où l'ironie permettrait un meilleur accès au moi et au monde. Néanmoins, il apparaît déjà que Maurice Barrès utilise l'ironie comme un levier qui doit mener le chroniqueur à dépasser les insuffisances du réel pour proposer au lecteur sa propre interprétation de celui-ci.

De même, et alors que l'on oppose souvent l'auteur du Roman de l'énergie nationale à celui du Culte $d u$ moi, la lecture du Voltaire dévoile l'origine précoce de certaines problématiques barrésiennes. Les voyages de Barrès en Bretagne l'amènent ainsi à formuler intuitivement les fondements d'une pensée nationaliste qui ne sera développée que quelques années plus tard. Les chroniques consacrées à cette région montrent très distinctement l'importance que Barrès accorde aux territoires français et à leurs arts. Si l'on ne peut pas encore parler de pensée politique structurée, certains extraits de la chronique sur la littérature de Basse-Bretagne laissent déjà entendre la voix des Déracinés. Cet article oppose deux formes de littérature: la littérature bretonne, jugée populaire et authentique, et celle développée par les poètes bretons installés à Paris. Barrès y souligne déjà le rôle et l'influence de la «terre» sur l'identité culturelle et littéraire d'un écrivain. Il relève ainsi le processus de déformation subi par les hommes de lettres qui résident à Paris, déformation qui est le résultat direct de l'éducation reçue au lycée dont Barrès dénoncera les dérives dans Les Déracinés à partir de l'année 1894.

Il est également possible d'observer qu'à l'époque du Voltaire, une unité se crée déjà autour de l'idée d'énergie. Présente dès ses premières chroniques, celle-ci devient de fait l'un des thèmes structurants de son œuvre romanesque. La lecture de la "Contagion des Rastignac» permet

5. M. Barrès, "Quinze cents métaphores», Le Voltaire, 23 janvier 1888. 
de faire le lien très tôt entre les deux premières trilogies de Barrès. En effet, dans la conception barrésienne du génie, le talent en littérature est rattaché à la maîtrise d'une énergie brute. L'article sur Balzac a pour sujet la mise en scène et l'incarnation de l'idée d'énergie, ainsi que les dérives qu'elle peut entraîner. Il évoque la fièvre, que Barrès appelle «napoléonite» ou «balzacienne», qui correspond à l'expression d'une énergie privée de direction. Si, en 1887, Barrès définit le terme dans une perspective égotiste, il pressent déjà les limites d'une telle conception. L'expérience brute et chaotique de l'énergie ne peut mener qu'à son propre épuisement. Tel est le constat final de Sous l'œil des barbares, que l'on retrouve dans l'article du Voltaire, et auquel Les Déracinés propose d'apporter une réponse. L'évolution de la pensée barrésienne vers la nécessité d'un retour à une conscience nationale plutôt qu'individualiste est ainsi perceptible, dès 1887, grâce aux problématiques soulevées par la question de l'énergie.

Le Voltaire apparaît par conséquent comme un laboratoire où se développe, par le biais d'une écriture expérimentale, la personnalité barrésienne. En un sens, l'œuvre romanesque de Barrès est déjà présente, en germe, dans ces chroniques. Miroir des pensées de Barrès, ces articles constituent une forme de Cahiers avant l'heure, qui dévoilent une écriture et une pensée littéraire dans leur phase d'élaboration. S'il est possible de considérer la presse, au cours des années de formation de Maurice Barrès, comme un laboratoire d'écriture barrésienne, il apparait toutefois que les liens qui rattachent la production romanesque du romancier de l'Énergie nationale aux articles de ce dernier tendent à évoluer avec les années. Ainsi, et bien que le journal demeure un espace d'expérimentation littéraire, les quotidiens avec lesquels collabore Maurice Barrès occupent de plus en plus la fonction de miroir. Ils renvoient en effet une image précise de l'univers romanesque barrésien, tout en participant à la diffusion de celui-ci: entre la fin des années 1880 et le début des années 1920, les quotidiens contribuent à la diffusion et à l'affirmation de l'imaginaire barrésien, de même qu'ils permettent de suivre l'évolution esthétique du romancier.

Dans ce cadre, si la collaboration de Maurice Barrès au Voltaire permet de comprendre l'origine de certaines thématiques romanesques, la lecture des articles de presse qui paraissent au cours des années suivante, et notamment pendant la période entre 1889 et 1905, amène à suivre l'évolution de l'écriture égotiste barrésienne vers le nationalisme. 


\section{La chronique barrésienne: de l'égotisme au nationalisme}

La période 1889-1905 est complexe car elle donne à voir une évolution esthétique et idéologique. Symbolisée par le passage d'une esthétique à une autre, elle semble aussi se traduire par un changement d'attitude face au monde. Néanmoins, comme Barrès l'explique lui-même, le passage de la pensée égotiste de l'homme libre à celle du romancier nationaliste n'est pas contradictoire:

Oui, L'Homme libre racontait une recherche sans donner de résultat, mais, cette conclusion suspendue, Les Déracinés la fournissent. Dans Les Déracinés, l'homme libre distingue et accepte son déterminisme, un candidat au nihilisme poursuit son apprentissage, et d'analyse en analyse, il éprouve le néant du Moi, jusqu’à prendre le sens social. La tradition retrouvée par l'analyse du moi, c'est la moralité que renfermait L'Homme libre, que Bourget réclamait et qu'allait prouver le roman de l'Énergie nationale 6.

La production journalistique de Barrès porte les traces, stylistiques et théoriques, de cette évolution esthétique qui place le moi barrésien au sein d'un collectif. S'il est vrai que la figure de Barrès prédomine dans chacune de ses chroniques, notamment du fait de l'omniprésence du «je» dans le texte, elle est parfois associée à un groupe par l'emploi du pronom "nous». Cette utilisation occasionnelle sous la plume de Barrès pose problème. Bien que l'emploi soit plutôt rare, en comparaison à l'omniprésence du «je», il est porteur de sens. Dans ses premiers articles, le "nous» désigne autant la famille des écrivains à laquelle Barrès considère appartenir que les cercles avant-gardistes qu'il souhaite approcher. Il est possible d'effectuer ce rapprochement grâce à l'étude du lectorat auquel s'adressent les petites revues dans lesquelles les articles de Barrès ont été publiés.

Toutefois, dans les textes qui suivent, la situation est souvent plus complexe. La position de Barrès au sein de la sphère littéraire a changé. Jeune écrivain à la position affirmée grâce au succès rencontré par ses premiers romans, il n'est plus en quête de l'appui des cénacles. Le "nous» auquel il fait allusion devient plus changeant et mouvant et fait de plus

6. M. Barrès, "À propos d'une réimpression de l'homme libre", Revue bleue, 10 septembre 1904. 
en plus souvent référence au peuple français. De même, le chroniqueur se réfère de plus en plus régulièrement à l'idée de patrimoine commun ancrant ainsi le «moi» dans une tradition:

Tant que les mêmes causes ont agi, nous avons eu les mêmes hommes, les mêmes événements. Tout ce siècle a vécu de la sensibilité balzacienne: des jeunes gens de la bourgeoisie, accourant pour l'ordinaire de province et avides de prendre leur part de pouvoir et de jouissance... ${ }^{7}$

Le moi barrésien, représenté par le "je» dans le texte, se voit alors enraciné au sein d'un moi collectif par l'entremise de la culture. C'est ainsi que les chroniques de société sur la littérature font état de ce phénomène. Dans un article publié en 1894, Barrès définit et caractérise l'influence qu'exerce Voltaire sur sa génération de la manière suivante:

L'importance qu'il [Voltaire] prend encore sur notre imagination, il la doit aux dictionnaires, aux récits des personnes âgées; mais de lui-même, il ne nous domine pas plus que ne le fait La Bruyère ou le chevalier de Grammont ${ }^{8}$.

Le but de la chronique barrésienne n'est plus alors de présenter un moi dénué de toute attache, mais de parvenir à le faire exister, sans lui ôter sa particularité, au sein d'un collectif, suivant en cela une trajectoire qui va du Culte du moi au Roman de l'énergie nationale et aux Scènes et doctrines du nationalisme. Il n'est pas question de nier la singularité de chacun dans la pensée barrésienne, mais de comprendre ce qui la constitue. La tradition, tout comme la persistance de valeurs communes, joue un rôle primordial dans la formation de celle-ci.

Oui, le bienfait dont nous remercions le maître qui vient de mourir, c'est qu'il a trouvé un joint pour conserver à l'esprit moderne le bénéfice de cette prodigieuse sensibilité catholique dont la plupart d'entre nous ne sauraient se passer, car elle a façonné trop longtemps nos ancêtres 9 .

Le passage par la littérature et la culture est dès lors nécessaire pour faire exister ce sentiment de cohésion. La chronique, par ses caractéristiques

7. M. Barrès, «Enfin Balzac a vieilli!», Le Journal, 16 février 1894.

8. M. Barrès, "Un grand homme du passé », Le Journal, 23 novembre 1894.

9. M. Barrès, «Renan", Le Figaro, 3 octobre 1892. 
génériques, permet à l'écrivain de s'essayer à une certaine forme d'écriture égotiste. Pourtant, au-delà de l'étude égotiste, Barrès inscrit sa réflexion dans une perspective communautaire. C'est ainsi que se dessine en creux, dans ses articles littéraires, les principes politiques de la théorie de la terre et des morts. Entre tradition et patriotisme, les chroniques barrésiennes participent à la formation et au développement de la voix du romancier nationaliste.

La confrontation des articles de presse et des romans de Barrès laisse toutefois entrevoir un autre axe d'unité au sein de l'œuvre de l'écrivain. Celui-ci se révèle à travers l'influence qu'exerce la poétique journalistique sur l'art romanesque de l'auteur du Culte du moi. En effet, si la presse connaît un phénomène de littérarisation, l'inverse est également vrai. La lecture des romans de Barrès montre en effet que l'écrivain a recours aux codes du journalisme pour répondre à son désir de rendre compte du monde qui l'entoure.

\section{Le roman-journal: poétique de l'art romanesque barrésien}

Les romans barrésiens, aussi divers soient-ils d'une trilogie à l'autre, semblent avoir été influencés par la poétique du journal. L'insertion de chroniques ou de textes de fiction, qui ont préalablement été publiés dans des quotidiens et des revues, est le premier signe de la présence matérielle de la presse au sein du roman barrésien. Les chroniques du Voltaire se révèlent très souvent être le texte original à partir duquel dérivent certains chapitres du Culte du moi. Comme l'a montré Pierre-Georges Castex dans son article "Barrès collaborateur du Voltaire» ${ }^{10}$, la partie "Paris à vingt ans», extraite du chapitre IV de Sous l'œil des Barbares, intègre des passages de la chronique "Hôpital pour les artistes" datée du 6 décembre $1886^{11}$. De même, la chronique "Une race tragique» ${ }^{12}$ constitue la base de travail du chapitre "Extase», issu du même ouvrage.

La présence du journal se fait encore plus explicite à mesure que l'on avance dans la lecture des romans de Barrès. Le projet romanesque barrésien rappelle en effet un désir de maîtriser le monde par la forme

Io. P.-G. Castex, «Barrès, collaborateur au Voltaire (1886-1888)».

II. M. Barrès , "Hôpital pour les artistes", Le Voltaire, 6 décembre 1887.

I2. M. Barrès, "Une race tragique», Le Voltaire, $1^{\text {er }}$ juin 1886. 
qui s'inspire du journal. La poétique romanesque barrésienne repose en effet sur une technique de collage qui met en relation des éléments qui apparaissent dissemblables par leur forme ou par leur portée. Intégrés à un projet romanesque plus large, ceux-ci sont toutefois unis par un ensemble d'éléments narratifs. Construire un «tout» cohérent, qui fédère des éléments qui divergent par leurs formes et le registre dont ils relèvent, semble en effet être l'un des principes de l'écriture romanesque barrésienne. La publication de certains articles sous forme de recueil, comme Du sang, de la volupté et de la mort, témoigne de la volonté barrésienne de rassembler des textes autonomes, qui constituent autant de facettes de la réalité, pour proposer une représentation totale du monde.

Cette façon de procéder rappelle la structure du journal. Un quotidien construit en effet une représentation de la réalité, à un instant déterminé, à partir d'éléments autonomes et disparates. Il se conçoit de fait comme un "tout", qui répond à un projet défini, mais qui serait subdivisé en différentes parties construites à l'image des rubriques journalistiques. Dans La littérature au quotidien ${ }^{13}$, Marie-Ève Thérenty explique que la naissance de la rubrique, sous la Monarchie de Juillet, répond à un besoin d'unité et de maîtrise du monde. L'étude du quotidien La Presse révèle en effet que les nouvelles sont alors publiées sans souci de créer des liens de causalité ou d'unité entre elles. La mise en page de ce périodique reflète le sentiment de fragmentation ressenti à l'époque. Face à celui-ci, la rubrique est censée permettre la création d'une unité au sein d'un univers médiatique disparate et morcelé. Elle permet une maîtrise et une organisation de l'information par thèmes. Les premiers romans de Barrès reproduisent en quelque sorte l'organisation du journal à travers la gestion de "rubriques romanesques». Entre un phénomène de littérarisation de la presse et la collaboration des écrivains journalistes aux quotidiens s'instaure un effet miroir. Outre l'association de textes différents au sein d'un même ensemble, c'est la nature même de ces textes qui permet d'établir une comparaison entre la structure du journal et celle du roman. Tout comme les quotidiens de l'époque, le roman barrésien se compose d'études de mœurs, de passages critiques et de lettres. Ainsi, les «méditations" sur Benjamin Constant et sur Sainte-Beuve présentes dans le cinquième chapitre d'Un homme libre rappellent les portraits littéraires et les critiques que Maurice Barrès publie dans les quotidiens

I3. M.-È. Thérenty, La littérature au quotidien, p. 80-82. 
de l'époque. De même, les chapitres consacrés aux voyages du narrateur (le chapitre VI «En Lorraine» et le chapitre VIII "À Lucerne, Marie B...») se font l'écho des récits de voyage qui paraissent dans la presse.

Ce choix structurel répond à la nécessité de comprendre le "moi» dans sa diversité. Un tel programme suppose la mise en place d'une écriture plurielle, capable de rendre compte des différentes étapes de la quête égotiste du narrateur et des différents mouvements de son «moi». À l'image du quotidien, dont la structure est subdivisée en rubriques, les deux premiers tomes du Culte $d u$ moi se définissent par une composition morcelée. Caractérisée par sa plasticité, l'écriture romanesque permet à l'écrivain de juxtaposer des textes qui appartiennent à des genres et à des registres différents. L'insertion de lettres, par exemple, est un procédé récurrent, présent dans des romans aussi différents qu'Un homme libre, Les Déracinés ou L'Appel au soldat.

Au-delà de la structure formelle des premiers romans de Barrès, l'influence de la presse se lit également dans l'affirmation positive d'un point de vue journalistique sur le monde. À travers ses romans, Maurice Barrès approche en effet l'art de l'écriture avec le regard du journaliste. Ce positionnement se traduit par la mise en place d'un art romanesque fondé sur le traitement fictionnel de l'actualité. La structure narrative du roman repose alors sur la mise en récit d'une actualité médiatique.

Le réalisme des Déracinés semble en effet parfois être issu du regard observateur du journaliste. Comme le montre Marie-Françoise Melmoux-Montaubin, l'écrivain-journaliste se pose en "penseur» et «en philosophe ${ }^{14}$. Elle explique que, pour celui-ci, «écrire le monde, ce n'est pas le représenter, conformément au principe de la mimésis, c'est aussi essayer d'en appréhender le sens, c'est prétendre promouvoir une interprétation " ${ }^{15}$. À l'image du chroniqueur, le narrateur entrecoupe son récit par des explications, des analyses et des commentaires qui mettent en avant l'interprétation barrésienne du monde. Au fil de sa trilogie, Barrès se propose de mettre à nu les raisons qui ont précipité la France dans la décadence. Le chapitre "La France dissociée et décérébrée» ${ }^{16}$, extrait des Déracinés, illustre parfaitement ce moment où la parole du

I4. M.-F. Melmoux-Montaubin L'écrivain-journaliste au XIXe siècle, p. 9.

I5. Ibid.

I6. M. Barrès, Les Déracinés, p. 616-620. 
journaliste rejoint celle du narrateur, dans une même volonté d'expliquer les mécanismes qui régissent la société de cette fin de siècle.

Plus précisément encore, le troisième tome du Roman de l'énergie nationale offre un exemple significatif de la façon dont le traitement de l'actualité, et par là même l'insertion du réel, est symptomatique de l'affirmation d'un point de vue journalistique au sein d'une ouvre romanesque. Ce roman, conçu à partir des notes prises par Maurice Barrès au Palais Bourbon, revient sur le scandale de Panama. Alors que Maurice Barrès décrit un monde parlementaire soumis à l'effervescence et à la pression médiatique, la narration de Leurs Figures suit l'actualité de cette affaire de corruption révélée par Édouard Drumont dans La Libre Parole. La particularité du roman de Barrès réside dès lors dans la façon dont le romancier utilise l'actualité du scandale pour construire son roman et dénoncer la décadence de la société de la Belle Époque.

Pour créer une "fiction d'actualité", la méthode du romancier consiste dès lors à intégrer des faits et des personnages ayant réellement existé au sein d'un univers romanesque déjà construit. Leurs Figures mêlent en effet étroitement l'univers romanesque barrésien, avec les personnages de Bouteiller et de François Sturel, et un quotidien médiatique connu des lecteurs de l'époque. La publication d'un de ses articles sur le scandale de Panama amène François Sturel à rencontrer Charles de Lesseps ${ }^{17}$. Le baron de Reinach, Georges Clemenceau, Édouard Ducret (directeur de La Cocarde au moment de la publication Leurs Figures) rejoignent l'univers romanesque de Barrès. La particularité de l'écriture barrésienne réside dans le fait que le romancier traite des évènements réels comme des faits d'actualité. Bien que l'écriture et la publication de Leurs figures soient postérieures au scandale de Panama, le roman s'appuie sur des notes prises à l'époque de ce dernier. Ceci donne l'impression de suivre l'affaire au quotidien telle qu'elle pourrait être relatée dans les quotidiens de l'époque. L'inscription du réel dans le roman barrésien renouvelle ainsi l'écriture romanesque. Ce n'est pas tant l'insertion de faits réels qui constitue une nouveauté, que leur traitement dans une perspective d'explication du monde. À partir de l'arrière-plan romanesque développé dans les deux premiers tomes du Roman de l'énergie nationale, qui constitue le cadre narratif de Leurs Figures, Barrès met en scène l'actualité de l'époque avec un véritable souci de réalisme et de précision. Il apparaît

17. M. Barrès, Les Déracinés, p. 1064. 
dès lors assez clairement que Barrès, à la manière d'un journaliste, utilise sa connaissance de l'actualité pour proposer une interprétation du monde qui lui est personnelle.

Outre la mise en scène de personnages réels, ce texte se caractérise par un glissement énonciatif qui déplace le cadre du roman vers les colonnes du journal. En effet, alors qu'il relate les stratagèmes du baron, le narrateur, à l'image du chroniqueur, construit son discours à partir de la complicité supposée qu'il partage avec son lecteur. La question se pose alors de savoir à quel lecteur il s'adresse. Le lecteur fictif du roman, dont la figure dépasse le contexte de production du livre, ou le lecteur de la Belle Époque qui suit les débats de La Libre Parole ou celui de $L a$ Cocarde? La remarque "Le baron lui fait dire que M. Christophle est menacé, ce que tout le monde savait à ce moment-là " ${ }^{18}$ et le constat "Ce n'est pas mal déjà qu'un personnage, à qui nos ministres demandent de diriger dans l'ombre et sans responsabilité les finances d'État, soit un bas filou piratant la Bourse au Palais-Bourbon " ${ }^{19}$ brouillent en effet les pistes. L'allusion faite à une information connue de tous, et plus encore la présence du possessif "nos", créent un cadre référentiel qui dépasse celui du roman. L'emploi de "nos» dans le texte romanesque abolit les frontières de la fiction et place le discours du narrateur au cœur d'un quotidien médiatique réel et connu des contemporains de Barrès.

La réussite de Maurice Barrès réside dans sa volonté de ne pas dissocier une forme d'«art de l'actuel» d'un projet romanesque plus ambitieux. Ainsi, ce n'est pas tant une certaine actualité que le romancier intègre à son roman qu'une façon de l'appréhender. En choisissant d'adopter un point de vue journalistique pour construire son roman, le romancier de l'Énergie nationale renouvelle la figure du journaliste et plus encore la perception qu'il est possible d'en avoir. Leurs Figures légitime en effet une façon de "sentir» et d'aborder le réel propre aux journalistes. Il ne s'agit plus uniquement de mettre en scène le journaliste en tant que type, selon une représentation élogieuse ou dépréciative, mais de révéler, dans le texte même, la dimension intemporelle et artistique du point de vue journalistique. Dans cette perspective, le journaliste se révèle artiste au même titre que le romancier ou le poète.

I8. M. Barrès, Les Déracinés, p. 1070.

19. Ibid. 
La lecture des articles de presse de Barrès permet de mettre en lumière l'unité d'une œuvre qui apparaît souvent comme éclatée ou scindée en plusieurs éléments. Grâce à l'éclairage offert par les études et les chroniques, il apparaît qu'au-delà des transferts thématiques qui rapprochent production journalistique et œuvre romanesque, c'est avant tout une façon d'apprécier le réel, en partie issue de la pratique du journalisme, qui unit les différents textes de l'écrivain. L'écriture barrésienne, qu'elle soit romanesque ou journalistique, se caractérise par une hybridité en mouvement qui capture le réel. Ainsi, dans une démarche formatrice et créative, le romancier suit les pas du chroniqueur, tandis que le journaliste s'inspire de l'écrivain. Et c'est dans la confrontation de ces points de vue qu'émerge l'unité de l'œuvre barrésienne.

Séverine Depoulain

Membre associé de l'unité de recherches Écritures EA 3943 de l'Université de Lorraine 


\section{BIBLIOGRAPHIE}

Textes

BArrès, Maurice, "Une race tragique», Le Voltaire, $1^{\text {er }}$ juin 1886.

—, "Hôpital pour les artistes", Le Voltaire, 6 décembre 1887.

—, Sous l'ail des barbares, in Le Culte du moi, Paris, Lemerre, 1888; Romans et voyages, éd. par Vital Rambaud, Paris, Laffont, «Bouquins», 1994.

—, Un homme libre, in Le Culte du moi, Paris, Perrin, 1891; Romans et voyages, éd. par Vital Rambaud, Paris, Laffont, "Bouquins», 1994.

—, Le Jardin de Bérénice, in Le Culte du moi, Paris, Perrin, 1891; Romans et voyages, éd. par Vital Rambaud, Paris, Laffont, "Bouquins», 1994.

—, Examen des trois idéologies, Paris, Perrin, 1892; Romans et voyages, éd. par Vital Rambaud, Paris, Laffont, «Bouquins», 1994.

—, «Renan», Le Figaro, 3 octobre 1892.

—, «Enfin Balzac a vieilli!», Le Journal, 16 février 1894.

—, «Un grand homme du passé», Le Journal, 23 novembre 1894.

—, L'Ennemi des lois, Paris, Perrin, 1893; Romans et voyages, éd. par Vital Rambaud, Paris, Laffont, "Bouquins», 1994.

-, Du sang, de la volupté et de la mort, in Le Culte du moi, Paris, Charpentier et Fasquelle, 1894; Romans et voyages, éd. par Vital Rambaud, Paris, Laffont, «Bouquins», 1994.

—, Les Déracinés, in Le Roman de l'énergie nationale, Paris, Charpentier et Fasquelle, 1897; Romans et voyages, éd. par Vital Rambaud, Paris, Laffont, "Bouquins», 1994.

-, L'Appel au soldat, in Le Roman de l'énergie nationale, Paris, Charpentier et Fasquelle, 1900; Romans et voyages, éd. par Vital Rambaud, Paris, Laffont, "Bouquins», 1994. 
—, Leurs Figures, in Le Roman de l'énergie nationale, Paris, Juven, 1902; Romans et voyages, éd. par Vital Rambaud, Paris, Laffont, «Bouquins», 1994.

—, Scènes et doctrines du nationalisme, Paris, Juven, 1902.

—, "À propos d'une réimpression de l'homme libre", Revue bleue, 10 (septembre 1904).

Girdardin, Emile, La Presse, 21 septembre 1836.

\section{Travaux}

Castex, Pierre-Georges, "Barrès, collaborateur au Voltaire (18861888) ", Maurice Barrès. Annales de l'Est, 1963, p. 51-61.

Davanture, Maurice, La jeunesse de Maurice Barrès (1862-1888), 2 vol., Paris, Champion, 1975.

Godo, Emmanuel, La légende de Venise, Lille, Presses Universitaires du Septentrion, 1996.

Melmoux-Montaubin, Marie-Françoise, L'écrivain-journaliste au XIX siècle: un mutant des lettres, Saint-Etienne, Cahiers intempestifs, «Lieux littéraires», 2003.

Thérenty, Marie-Ève La littérature au quotidien. Poétiques journalistiques au XIX $X^{\mathrm{e}}$ siècle, Paris, Seuil, «Poétique», 2007. 
